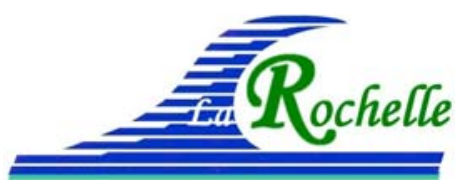

XVèmes Journées Nationales Génie Côtier - Génie Civil

La Rochelle, 29 au 31 mai 2018

DOI:10.5150/jngcgc.2018.084 @ Editions Paralia CFL

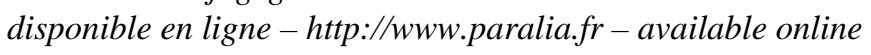

\title{
Analyse statistique du lien entre les variables d'aléa et les dommages par submersion
}

\author{
Florian ARNOUX ${ }^{1,2}$, Stéphane ABADIE ${ }^{1}$, Ivan KOJADINOVIC ${ }^{2}$
}

1. Laboratoire SIAME, EA4581, Fédération MIRA FR2152, Université de Pau et des

Pays de l'Adour, Allée du parc Montaury, 64600 Anglet, France.

stephane.abadie@univ-pau.fr

2. LMAP, UMR 5142, Fédération MIRA FR2152, Université de Pau et des Pays de

l'Adour, Avenue de l'Université, BP1155, 64013 Pau, France.

\section{Résumé :}

Une base de données de niveau de dommages consécutif à la submersion couvrant les 60 dernières années et focalisée sur la Grande Plage de Biarritz a été récemment élaborée. Cette base repose sur l'analyse d'archives et de documents de presse locaux. Parallèlement, une base de données de variables d'aléas couvrant la même période a également été constituée. Elle utilise des simulations numériques pour les vagues et une analyse harmonique pour les niveaux d'eau, validées par les observations disponibles sur site. Dans le présent article, nous détaillons l'analyse du lien statistique existant entre les deux bases de données. L'objectif est de trouver quelles variables d'aléa ou combinaison de variables expliquent le mieux l'occurrence de la submersion de la Grande Plage et la survenue de dommage. Les résultats préliminaires montrent que le couple flux maximum d'énergie des vagues et niveau d'eau maximum durant l'événement expliquent très bien les dommages répertoriés sur cette plage spécifique.

\section{Mots-clés :}

Submersion, Biarritz, Indicateurs, Statistiques, Dommages, Données historiques.

\section{Introduction}

Le programme MAREA pour "Modélisation et Aide à la décision face aux Risques côtiers en Euskal Atlantique", est un projet de recherche INTERREG POCTEFA, visant à mieux comprendre les épisodes de tempêtes sur la côte basque afin de prévoir les risques de submersion marine et d'érosion côtière grâce à la mise en place d'outils d'alerte innovants. Un des objectifs du programme est le développement d'indicateurs de danger permettant de quantifier le niveau de dommage attendu en fonction des paramètres de l'aléa. La communication que nous proposons ici, réalisée dans le cadre de ce programme, est focalisée sur le site de la Grande Plage à Biarritz. Nous cherchons à déterminer quelle variable d'aléa ou combinaison de variables d'aléas explique le mieux les données d'impact observées sur ce site spécifique. 


\section{Thème 7 - Risques côtiers}

\section{Méthode}

\subsection{Bases de données}

\subsubsection{Données d'impact à la côte}

Une base de données (ABADIE et al., 2018), collectée par une recherche dans les archives sur la période 1950-2014, a été réalisée afin de connaître les événements historiques des inondations côtières le long de la côte basque. Les principales sources de données proviennent d'une recherche systématique dans la presse nationale et locale, en particulier le journal régional Sud-Ouest, ainsi que dans les archives publiques tenues par les représentants du gouvernement, les organismes publics et les autorités locales. La méthodologie s'est inspirée du projet français COCORISCO (HENAFF \& PHILIPPE, 2014) sur les risques côtiers et s'est appuyée sur les expériences antérieures de recherche et de cartographie des archives historiques sur les catastrophes menées par une partie de l'équipe (BOUISSET et al., 2003). Une évaluation de la probabilité de submersion et des dommages connexes a été effectuée. Pour cela, nous nous sommes concentrés sur la région de la Grande Plage à Biarritz pour laquelle les séries d'informations les plus continues ont été collectées. Nous avons essayé de trouver des preuves de submersion ou de non-submersion. Les événements qui ont provoqué des submersions à la Grande Plage ont été marqués par un indice 1 et 0 en cas de nonsubmersion. Un indice de confiance (1: fiable, 0 : faible fiabilité) a été ajouté à cette information pour un traitement statistique plus pertinent. La même analyse a été effectuée pour l'intensité des dommages. Une échelle à trois niveaux (2 pour les dommages significatifs, 1 pour les dommages modérés et 0 pour les dommages nuls) a été choisie comme étant la plus appropriée aux données. Un indice de confiance sur cette valeur a également été ajouté pour le traitement ultérieur. Des renseignements indiquant si l'événement était classé ou non dans la catégorie catastrophe naturelle (CATNAT) et si, pendant la tempête, des mesures de protection (e.g., la construction d'un brise-lames temporaire devant le casino) ont été prises ont également été ajouté pour compléter cette base de données.

Le nombre de tempêtes, pour lesquelles seule Biarritz a été citée, est de 30 et le nombre de submersion à la Grande plage est de 13, ce qui représente un tiers des tempêtes observées sur la période 1950-2014. Parmi ces submersions, seules 2 sont considérées comme non fiables. En ce qui concerne les événements de non-submersion, l'information est la plupart du temps considérée comme non fiable. Dans 2 événements seulement, la submersion est exclue avec confiance. Ces deux événements sont aussi importants que ceux impliquant la submersion puisqu'ils peuvent servir de référence pour tester des combinaisons de paramètres d'aléa pour lesquelles il n'y a pas submersion alors que l'on pourrait s'attendre au contraire. Ainsi, ce genre de cas peut aider à situer la limite entre submersion et non-submersion. En ce qui concerne les 


\section{XVèmes Journées Nationales Génie Côtier - Génie Civil \\ La Rochelle, 29 au 31 mai 2018}

dommages causés par la submersion, 5 événements ont généré des dommages de niveau 2 avec une bonne fiabilité et 4 ont causé des dommages modérés avec une seule évaluation jugée non fiable dans cette catégorie. Les tempêtes restantes n'ont pas causé de dégâts avec un bon niveau de confiance. Les tempêtes qui ont causé les principaux dommages (niveau 2) suite à une submersion sont les tempêtes du 28-29 décembre 1951, du 9-10 novembre 2010 et les récents événements de l'hiver 2013-2014: c'est à dire les tempêtes Hercules (4-7 janvier), Nadja (1-2 février) et Christine (3-5 mars).

\subsubsection{Données d'aléa}

Parallèlement à cette base de données de dommage, une base de données d'aléa couvrant la même période a également été constituée (ARNOUX et al., 2018). Elle est composée des données de vagues et de niveau d'eau.

Les données de niveaux d'eau sont obtenues par les observations au marégraphe de Socoa. Comme il y a beaucoup de données manquantes et que les observations ne couvrent pas l'ensemble de la période considérée, une reconstruction du signal a été effectuée. Ce modèle fait la somme de trois composantes: le niveau moyen de l'eau au temps $\mathrm{t}$, qui est une moyenne mobile calculée à partir des moyennes mensuelles des mesures, le signal de marée à l'instant $t$, calculée par analyse harmonique des observations du marégraphe, et une estimation grossière de la surcote atmosphérique au temps $\mathrm{t}$, obtenue à partir de données de simulation de la pression atmosphérique. La dernière partie du modèle pourrait être discutable puisqu'elle ne prend pas en compte le vent. Néanmoins, ce modèle assez simple semble suffisant pour obtenir des séries précises de valeurs de niveau d'eau pour la période 1949-2015, ce que montre la comparaison avec le signal du marégraphe. D'autre part, il semble que ce niveau d'eau soit assez homogène spatialement comme le montre les comparaisons des marégraphes de Socoa et de Boucau.

Les observations des vagues étant également limitées spatialement et temporellement, les données de vagues utilisées dans ce travail, ont été obtenues par des simulations numériques. La simulation principale, appelée WWMII, couvre la période d'étude (1949 à 2015). Elle a été réalisé à l'aide d'un système de modélisation qui couple le modèle de circulation 2DH basé sur SELFE (ZHANG et al., 2008) et le modèle spectral WWMII2 pour les vagues (ROLAND, 2008). La configuration décrite dans BERTIN et al. (2015) a été mise en œuvre sur l'ensemble de l'océan Atlantique Nord, avec une résolution spatiale de quelques dizaines de $\mathrm{km}$ dans l'océan profond à $1 \mathrm{~km}$ le long de la côte du golfe de Gascogne. Ce modèle a été forcé avec les champs de vent et de pression sous-jacents provenant de la réanalyse NCEP/NCAR (KALNAY, 1996), assurant ainsi une bonne homogénéité temporelle sur la période étudiée. Le modèle donne accès aux paramètres de vagues intégrés (i.e., hauteur significative $\mathrm{H}_{\mathrm{m} 0}$, etc) à quelques points correspondant aux mesures ou devant les principales villes côtières basques (à $-100 \mathrm{~m}$ et $-15 \mathrm{~m}$ de profondeur). Des comparaisons ont également été 


\section{Thème 7 - Risques côtiers}

effectuées avec la base de données HOMERE obtenue à partir du modèle spectral WAVEWATCH III et une grille non structurée couvrant le golfe de Gascogne (ROLAND, 2008).

Les données de WWMII sont comparées aux mesures du houlographe directionnel qui se trouve à environ $6 \mathrm{~km}$ au large de Biarritz sur la période de mesure (à partir de 2009). On trouve une erreur RMSE de $48 \mathrm{~cm}$, un coefficient de détermination R2 de 0.73 et un biais de $6.98 \%$. L'analyse des graphes quantile/quantile montre une sous-estimation du modèle systématique pour les états de mer extrême, préjudiciable pour ce type d'étude justement focalisé sur ce type d'événement. Dans ARNOUX et al. (2018), une correction quantile empirique est appliquée aux données pour tenter de corriger ce biais. C'est cette dernière donnée corrigée qui considérée dans le présent travail. Le lecteur intéressé pourra se référer à ARNOUX et al. (2018) pour plus de précision sur ce sujet.

\subsection{Variables agrégées}

Afin d'obtenir des variables prédictives pertinentes, on peut construire des variables dites agrégées à partir des variables d'aléa de base que sont les niveaux d'eau et les paramètres intégrés de vagues. Dans ce travail, nous avons ainsi testé en plus des variables de base, le flux d'énergie des vagues qui permet d'agréger hauteur de vague et période énergétique, cette dernière intervenant dans le calcul de la vitesse de groupe $C_{g}$. Le flux d'énergie se calcule par la formule suivante: $\mathrm{F}=\left(\rho \mathrm{gH}_{m 0}^{2} C_{g}\right) / 16$, avec $\rho$ la masse volumique de l'eau de mer et $g$ la constante de gravitation.

Le Run-up $\mathrm{R}$ est la hauteur atteinte par l'eau après déferlement d'une vague. Ce paramètre peut facilement s'additionner au niveau d'eau de manière à représenter physiquement les hauteurs atteintes par un état de mer donné. De plus, il est assez facile de déterminer un seuil réel (mur, haut d'une plage) représentant la limite à franchir par le Run-up pour être en présence d'un événement de submersion. STOCKDON et al. (2006) donnent la formule empirique suivante permettant de prédire la valeur de run-up dépassée seulement par $2 \%$ des vagues de l'état de mer étudié en différenciant les contributions respectives du set-up (i.e., élévation moyenne du plan d'eau due à la dissipation dans le déferlement), du jet de rive des vagues incidentes et des vagues infragravitaires dont on sait qu'elles représentent une contribution très importante pour la submersion sur la portion de côte étudiée :

$$
R_{2 \%}=1.1 *\left(0.35 \beta \sqrt{H_{m 0} \frac{\mathrm{gT}_{p}^{2}}{2 \pi}}+\sqrt{\frac{1}{2} H_{m 0} \frac{\mathrm{gT}_{p}^{2}}{2 \pi}\left(0.563 \beta^{2}+0.004\right)}\right)
$$

où $T_{p}$ est la période pic pour un état de mer donné et $\beta$ est la pente moyenne de la plage étudiée dans la partie concernée par le run-up.

\subsection{Méthode de tests statistiques}




\section{XVèmes Journées Nationales Génie Côtier - Génie Civil \\ La Rochelle, 29 au 31 mai 2018}

Dans la base de données d'impact à la côte, chaque événement est représenté par une date de début et une date de fin ainsi que par deux indices qualitatifs (eux-mêmes complétés par des indices de confiance). Afin de relier les données d'aléas à ces données, chaque variable a été extraite pour les dates d'événements considérés.

Pour chaque variable, les moyennes et maximums de chaque événement sont ensuite extraits.

Cette base de données peut alors être étudiée par différentes approches.

Il est par exemple possible d'essayer de relier les variables des deux bases de données par une analyse en composante principale (ACP). Dans une $\mathrm{ACP}$, on part d'un tableau à $p$ variables et $n$ individus. L'objectif est de remplacer les variables initiales corrélées par de nouvelles variables, non corrélées, appelées composantes principales qui sont des combinaisons linéaires des variables initiales. Le nuage des individus, dans ce nouvel espace voit sa variabilité maximisée.

Une autre méthode plus empirique consiste à tester des indicateurs plus ou moins physiques basés sur les variables agrégées par exemple ou des analyses multivariées dont l'objectif est d'expliquer au mieux les dommages observés.

\section{Résultats}

Les événements recensés par la base de données de dommages sont résumés dans le tableau 1.

Tableau 1. Base de données d'impact sur la Grande Plage de Biarritz. 


\section{Thème 7 - Risques côtiers}

\begin{tabular}{|c|c|c|c|c|c|c|}
\hline Tempête & Début & Fin & Submersion & Confiance & Dommages & Confiance \\
\hline 1 & $28 / 12 / 1951$ & $30 / 12 / 1951$ & 1 & 1 & 2 & 1 \\
\hline 2 & $14 / 12 / 1958$ & $15 / 12 / 1958$ & 0 & 0 & 0 & 1 \\
\hline 3 & $12 / 11 / 1961$ & $14 / 11 / 1961$ & 0 & 0 & 0 & 1 \\
\hline 4 & $17 / 01 / 1965$ & $20 / 01 / 1965$ & 1 & 1 & 1 & 1 \\
\hline 5 & $30 / 10 / 1967$ & $05 / 11 / 1967$ & 1 & 1 & 1 & 0 \\
\hline 6 & $11 / 12 / 1968$ & $17 / 12 / 1968$ & 1 & 1 & 0 & 0 \\
\hline 7 & $20 / 01 / 1972$ & $22 / 01 / 1972$ & 1 & 0 & 0 & 1 \\
\hline 8 & $06 / 02 / 1974$ & $08 / 02 / 1974$ & 1 & 0 & 0 & 1 \\
\hline 9 & $24 / 01 / 1984$ & $25 / 01 / 1984$ & 0 & 0 & 0 & 1 \\
\hline 10 & $30 / 01 / 1988$ & $01 / 02 / 1988$ & 0 & 0 & 0 & 1 \\
\hline 11 & $25 / 02 / 1989$ & $27 / 02 / 1989$ & 0 & 0 & 0 & 1 \\
\hline 12 & $30 / 01 / 1990$ & $01 / 02 / 1990$ & 1 & 1 & 1 & 1 \\
\hline 13 & $07 / 02 / 1996$ & $09 / 02 / 1996$ & 0 & 0 & 0 & 1 \\
\hline 14 & $28 / 12 / 1998$ & $02 / 01 / 1999$ & 1 & 1 & 1 & 1 \\
\hline $15=$ Martin & $27 / 12 / 1999$ & $31 / 12 / 1999$ & 0 & 1 & 0 & 1 \\
\hline 16 & $06 / 11 / 2000$ & $10 / 11 / 2000$ & 0 & 0 & 0 & 1 \\
\hline 17 & $07 / 12 / 2000$ & $09 / 12 / 2000$ & 0 & 1 & 0 & 1 \\
\hline 18 & $14 / 08 / 2008$ & $16 / 08 / 2008$ & 0 & 0 & 0 & 1 \\
\hline $19=$ Klaus & $23 / 01 / 2009$ & $25 / 01 / 2009$ & 0 & 0 & 0 & 1 \\
\hline 20 & $09 / 11 / 2010$ & $11 / 11 / 2010$ & 1 & 1 & 2 & 1 \\
\hline $21=$ Quirin & $15 / 02 / 2011$ & $17 / 02 / 2011$ & 0 & 0 & 0 & 1 \\
\hline 22 & $18 / 02 / 2011$ & $23 / 02 / 2011$ & 1 & 1 & 0 & 1 \\
\hline 23 & $13 / 12 / 2011$ & $18 / 12 / 2011$ & 0 & 0 & 0 & 1 \\
\hline 24 & $26 / 01 / 2013$ & $31 / 01 / 2013$ & 0 & 1 & 0 & 1 \\
\hline 25 & $09 / 02 / 2013$ & $14 / 02 / 2013$ & 0 & 0 & 0 & 1 \\
\hline $26=$ Dirk & $23 / 12 / 2013$ & $26 / 12 / 2013$ & 0 & 0 & 0 & 1 \\
\hline $27=$ Hercules & $04 / 01 / 2014$ & $08 / 01 / 2014$ & 1 & 1 & 2 & 1 \\
\hline $28=$ Nadja & $01 / 02 / 2014$ & $03 / 02 / 2014$ & 1 & 1 & 2 & 1 \\
\hline $29=$ Andrea & $26 / 02 / 2014$ & $03 / 03 / 2014$ & 0 & 0 & 0 & 1 \\
\hline $30=$ Christine & $03 / 03 / 2014$ & $06 / 03 / 2014$ & 1 & 1 & 2 & 1 \\
\hline
\end{tabular}

Les dates de début et de fin sont utilisées pour extraire de la base de données d'aléas les caractéristiques de chaque événement. Les maxima et moyennes de chaque variable ont été choisis pour définir chaque événement. Il a également été décidé de prendre en compte la durée de l'événement. Cette variable sera biaisée vu son mode d'obtention et contrainte par la qualité des données. Il faut noter qu'un vecteur de données correspond à chaque événement, ce qui donne un tableau de 30 lignes.

Pour réduire le nombre de variables d'aléa et de variables agrégées et proposer facilement un indicateur de dégâts, une ACP est réalisée sur toutes les variables. $79.5 \%$ de la variabilité est expliquée en gardant 2 composantes principales. L'axe 1 explique $64 \%$ de variabilité et est très corrélé avec toutes les variables liées aux états de mer (moyennes et maximales) tandis que le second axe est corrélé à la durée et au niveau d'eau. Les individus représentés sur ce plan factoriel montrent une bonne distinction en termes de dégâts observés notamment sur l'axe 1. Cette ACP est néanmoins peu informative et les axes ne semblent pas avoir une interprétation physique évidente.

Dans la suite, les événements sont étudiés relativement à des paires de variables ou à des variables agrégées. Pour chaque événement, le maximum du flux et le niveau d'eau correspondant sont calculés. Les événements responsables de dégâts se distinguent bien selon la variable Flux mais le niveau d'eau au même moment ne semble pas forcément important. De plus, il est difficile de différencier les événements sur ce plan. 


\section{XVèmes Journées Nationales Génie Côtier - Génie Civil \\ La Rochelle, 29 au 31 mai 2018}

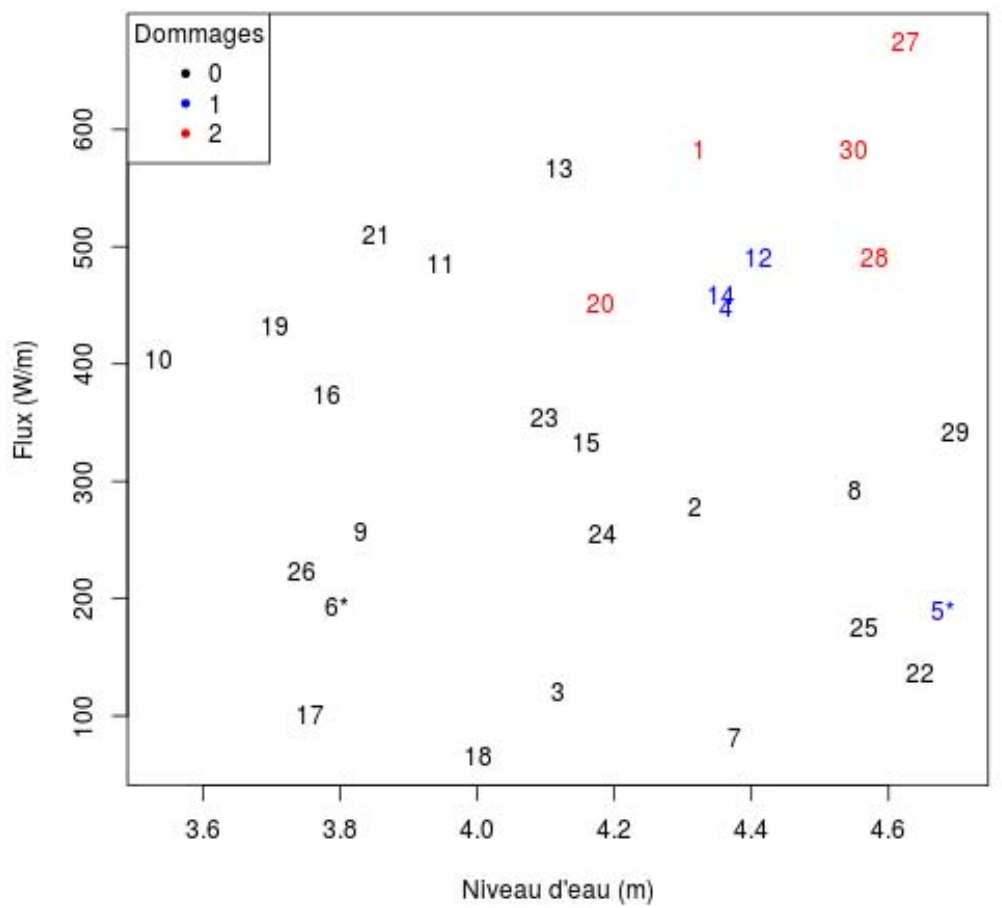

Figure 1. Événements de tempête sur la Grande Plage de Biarritz classé dans un plan associant le maximum du flux et la hauteur d'eau.

$\mathrm{Si}$ on prend en compte le niveau d'eau maximum et le flux correspondant, la classification est légèrement plus pertinente car le flux varie moins que le niveau d'eau lors d'un événement de tempête. Les problèmes précédents sont néanmoins toujours présents. On peut également, pour chaque événement, calculer le niveau maximum et le flux maximum (les deux maximums n'ayant pas forcément lieu au même moment). Les événements avec les indices de dégât 1 et 2 sont regroupés dans le coin supérieur droit du graphe (figure 1). Les maxima des deux variables permettent de bien distinguer les événements destructifs des autres. Néanmoins, sur la durée de chaque événement, les deux maxima n'ont aucune raison d'intervenir au même moment, ce que l'on observe dans les données. Ainsi, même si ce critère semble donner de bons résultats, il est un peu délicat à justifier physiquement.

Pour la figure 2, les valeurs maximales, de la somme entre le Run-up et le niveau d'eau sont représentés pour chaque événement. Il y a plusieurs événements n'entraînant pas de dommages qui apparaissent parmi les événements ayant conduit à des destructions. 


\section{Thème 7 - Risques côtiers}

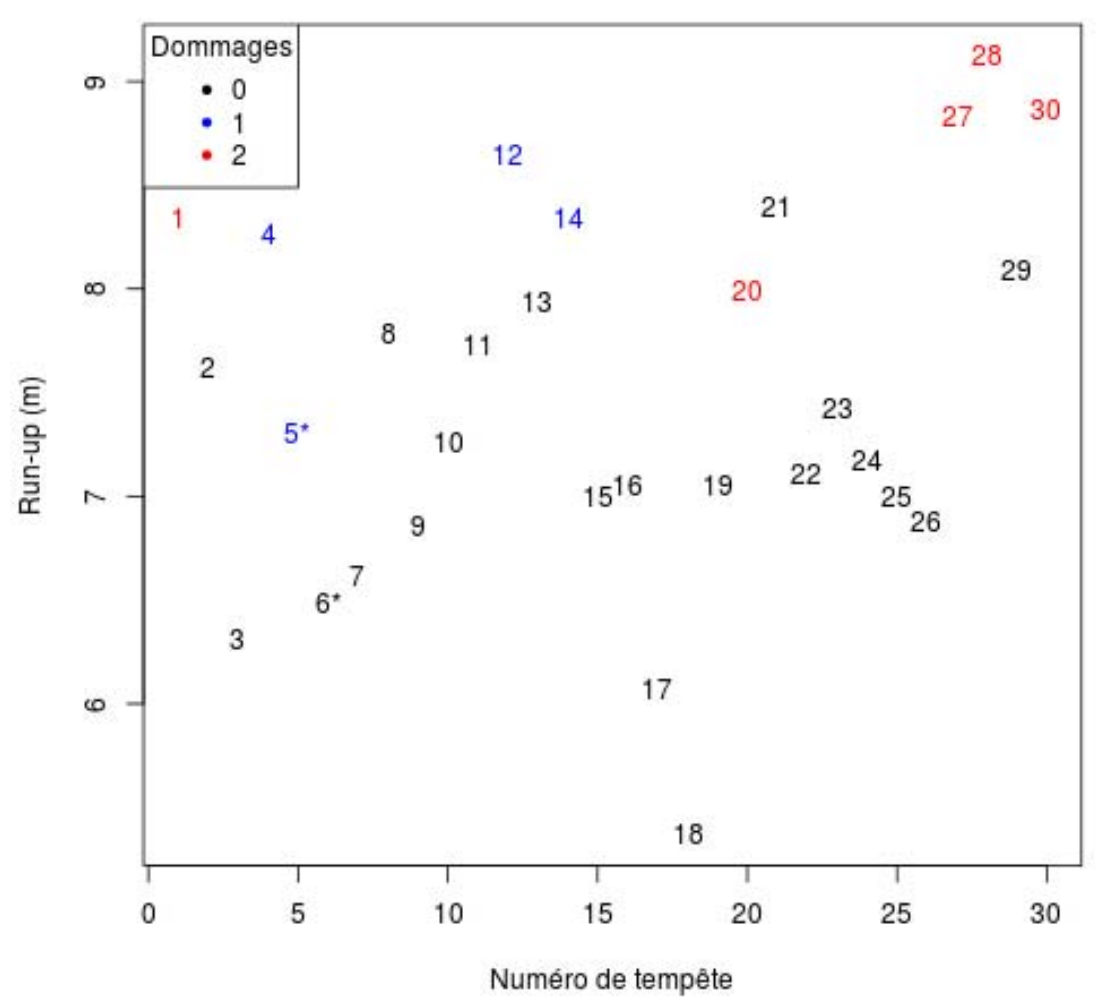

Figure 2. Valeur du run-up maximum calculé pour chaque événement de tempête sur la Grande Plage de Biarritz

\section{Discussion}

La mise en commun de ces deux bases de données permet de lier facilement les dégâts observés aux données de l'océan. La meilleure manière de distinguer les événements destructeurs semble être de tenir compte du niveau d'eau maximum et du maximum du flux de l'événement. Néanmoins la limitation du jeu de données et les réserves que l'on peut émettre quant à sa fiabilité invitent à rester prudent sur l'utilisation d'une telle fonction de discrimination. Il faut également rappeler que la base de données d'aléas présente le souci des données issues de simulations numériques présentant des corrections statistiques.

La tempête 5 (Novembre 1967) qui apparaît en bleu (*) dans le jeu de données (dégâts de niveau 1) se situe dans nos analyses en permanence parmi d'autre tempête n'ayant générées aucun dégât. Si on se réfère au tableau des tempêtes, c'est la seule pour laquelle le niveau de dégâts est peu fiable, ce qui paraît cohérent. A l'inverse les tempêtes 13 (Février 1996), 21 (Quirin, Février 2011) et 29 (Andréa, Fin Février 2014), 


\section{XVèmes Journées Nationales Génie Côtier - Génie Civil \\ La Rochelle, 29 au 31 mai 2018}

apparaissent dans des zones regroupant des tempêtes dévastatrices (figures 1 et 2) alors qu'a priori elles n'auraient pas générés de dégâts. Le 0 dégât associé à ces tempêtes repose sur la présence de descriptions de l'événement dans les archives ou la presse mais l'absence de mention de dégâts spécifiques. L'hypothèse suivant laquelle cela signifie automatiquement qu'aucun dégât ne s'est produit est peut-être discutable. Pour la 3ème tempête (Andrea), en plus de la remarque précédente, il faut ajouter que cette tempête s'est produite durant l'hiver 2014 après une succession de tempêtes dévastatrices et que des solutions de protection avaient été mises en place pour contrer cet événement spécifique ce qui peut biaiser l'analyse.

Enfin, dans le tableau 1, on remarque que l'échantillonnage des tempêtes obtenu dans ce tableau n'est pas homogène en temps. On observe clairement un nombre plus important de tempêtes dans les années 2010 (12) alors qu'elles en général entre 2 et 4 durant les décennies précédentes. Cette sur-représentation, durant la dernière période, peut s'expliquer physiquement par l'occurrence d'un hiver tout à fait anormal à l'échelle des 50 dernières années et par un léger biais dans la base de données de dommage pour laquelle il a été plus aisé de faire des recherche dans la période la plus récente.

\section{Conclusions et perspectives}

Dans ce travail, nous avons tenté de relier les paramètres de vagues et de niveau d'eau avec les dommages observés sur la Grande Plage de Biarritz. Les résultats préliminaires montrent que le flux maximum d'énergie des vagues et le niveau d'eau maximum durant l'événement forment un couple pertinent pour la quantification des dommages consécutifs à la submersion. D'autres indicateurs ayant potentiellement une meilleure interprétation physique, seront testés dans l'avenir tel que le cumul du flux d'énergie audessus d'un certain niveau d'eau sur une durée donnée par exemple.

6. Remerciements : Ce travail est financé en partie par le programme européen POCTEFA MAREA EFA046/15. Les auteurs remercient l'Université de Pau et des Pays de l'Adour et le Conseil Départemental 64 pour leur soutien financier à la bourse de doctorat de Florian Arnoux ainsi que les autorités locales pour leur aide dans la collecte des données.

\section{Références bibliographiques}

ABADIE S., BEAUVIVRE M., EGURROLA E., BOUISSET C., DEGREMONT I., ARNOUX F. (2018). A database of recent historical storm impact on the French Basque coast.). Proceedings from the $15^{\text {th }}$ International Coastal Symposium (ICS) 2018 (Busan, Republic of Korea). Journal of Coastal Research, Special Issue.

ARNOUX F., ABADIE S., BERTIN X., KOJADINOVIC I. (2018). A database to study storm impact statistics along the Basque Coast. Proceedings from the $15^{\text {th }}$ International 


\section{Thème 7 - Risques côtiers}

Coastal Symposium (ICS) 2018 (Busan, Republic of Korea). Journal of Coastal Research, Special Issue.

BERTIN X., LI K., ROLAND A., BIDLOT J.-R. (2015). The contribution of short waves in storm surges: two case studies in the Bay of Biscay. Continental Shelf Research, Vol. 96, pp 1-15. https://doi.org/10.1016/j.csr.2015.01.005

BOUISSET C., DEGREMONT I., PUYO J-Y. (2003). Contribution à la cartographie historique des phénomènes naturels à risque dans les Hautes-Pyrénées, Laboratoire SET, Univ. Pau \& Pays Adour, Rapport Technique, 32 p.

HENAFF A. (ed), PHILIPPE, M. (2014). Gestion des risques d'érosion et de submersion marine, Guide méthodologique. Projet Cocorisco. 156 p.

KALNAY E. et al. (1996). The NCEP/ NCAR reanalysis project. Bul. of the American Met. Soc. 77, 437-471. https://doi.org/10.1175/1520-0477(1996)077<0437:TNYRP >2.0.CO;2

ROLAND A. (2008). Development of WWM II: Spectral wave modelling on unstructured meshes. Ph. D. thesis, Inst. of Hydraul. and Wave Resour. Eng., Techn. Univer. Darmstadt, Germany.

STOCKDON H.F., HOLMAN R.A., HOWD P.A., SALLENGER JR A.H. (2006). Empirical parameterization of setup, swash, and runup. Coastal Engineering, Vol. 53(7), pp 573-588. https://doi.org/10.1016/j.coastaleng.2005.12.005 